\title{
Injúria orgânica da circulação extracorpórea nos três primeiros meses de vida
}

\author{
Décio O. ELIAS*, Maria Helena L. SOUZA*, Bernardo S. LACERDA*, Francisco Eduardo S. FAGUNDES*, \\ Francisco J. S. LINO*, Márcio TIRABOSHI*
}

RBCCV 44205-101

ELIAS, D. O.; SOUZA. M. H. L.; LACERDA, B. S.; FAGUNDES, F. E. S.; LINO, F. J. S.; TIRABOSHI, M. - Injúria orgânica da circulaçăo extracorpórea nos três primeiros meses de vida. Rev. Bras. Cir. Cardiovasc., 5(1): 1-8, 1990.

RESUMO: Diversos fatores contribuiram para aumentar o número de procedimentos com circulaçăo extracopórea para neonatos e lactentes. Esses pacientes necessitam procedimentos especiais de perfusăo, fundamentos em sua fisiologia particular, nas características do seu metabolismo e na sua resposta aos desvios da homeostase, principalmente a acidose lática. Sáo analisados os protocolos de perfusăo em 110 procedimentos com parada circulatória total, para a realizaçăo da correçăo intracardiaca, durante a qual diversos mecanismos de injúria ao sangue foram identificados. A injúria mecânica, por açăo das forças desenvolvidas na circulação artificial do sangue e nas trocas gasosas, pode danificar os elementos figurados e as proteinas do plasma sangüineo. O contato com as superfícies do circuito extracorpóreo pode ativar os sistemas das cininas, da coagulaçăo, da fibrinolise e do complemento, por ativaçáo inicial do fator XII e produzir uma reaçăo inflamatória generalizada. A produçăo de ácido lático pode modificar o pH ideal para a funçáo enzimática e celular, e o superaquecimento do sangue, durante o reaquecimento, pode produzir dano ao sangue semelhante àquele causado pelos fatores físicos da circulação extracorpórea. A perfusăo neonatal e de pequenos lactentes é um continuado exercício de atençăo aos detalhes e respeito aos aspectos particulares da fisiologia, do metabolismo e da resposta à injúria.

DESCRITORES: circulação extracorpórea, crianças.

\section{INTRODUÇĀO}

Por longo tempo, a perfusão para crianças consistia apenas em uma miniaturização dos equipamentos e dos protocolos utilizados para os adultos.

A introduçăo da hipotermia profunda e da parada circulatória total, ao final dos anos 60 , contudo, permitiram melhor individualizaçăo e desenvolvimento da circulaçāo extracorpórea (CEC) infantil ${ }^{2}$ 9, 12, 16.

A aceitaçăo da ecocardiografia bidimensional, como método de eleiçăo para rastreamento e diagnóstico de cardiopatias em neonatos e pequenos lactentes, bem como a criação de centros especializados, contribuíram para $o$ aumento do número de crianças de baixo peso submetidas a cirurgia corretiva com circulação extracorpórea.

O impulso mais recente, nesse sentido, foi a ampla adoçăo da operaçăo de Jatene, como uma nova opção de tratamento para a forma simples de transposiçăo das grandes artérias, nas duas primeiras semanas de vida ${ }^{1}$. 22, 27, 29

A experiência tem, portanto, demonstrado que, cada vez mais, teremos número crescente de crianças de bai-

Trabalho realizado no Hospital de Cardiologia de Laranjeiras. Rio de Janeiro, RJ, Brasil.

Apresentado ao 17: Congresso Nacional de Cirurgia Cardiaca. Belo Horizonte, MG, 6 e 7 de abril, 1990.

- Do Hospital de Cardiologia de Laranjeiras.

Endereço para separatas: Décio O. Elias. Rua Roberto Oswaldo Cruz, 254. 22750 Rio de Janeiro, RJ, Brasil. 
ELIAS, D. O.; SOUZA, M. H. L.; LACERDA, B. S.; FAGUNDES, F. E. S.; LINO, F. J. S.; TIRABOSHI, M. - Injúria orgânica da circulaçăo extracorpórea nos três primeiros meses de vida. Rev. Bras. Cir. Cardiovasc., 5(1): 1-8, 1990.

xa idade e peso corporal submetidas a procedimentos com circulação extracorpórea, para os quais são necessários técnicas e protocolos que produzam o mínimo de alteraçōes, face à pequena margem de tolerância aos grandes desvios da fisiologia, característica primordial desse grupo de pacientes ${ }^{4}$.

No presente trabalho, vamos confinar nossas consideraçōes às técnicas e protocolos adotados para neonatos e pequenos lactentes, com peso corporal inferior a $8 \mathrm{~kg}$, e os riscos de injúria orgânica severa, identificados ao longo de uma experiência com 190 procedimentos, em crianças de baixo peso.

A maioria das injúrias e as complicaçōes que podem determinar são mais freqüentes e significativas nas crianças com idade inferior a três meses e formam a base de nossa discussão ${ }^{4,8,20,26}$.

\section{CASUÍSTICA E MÉTODOS}

Analisamos as técnicas empregadas em 110 pacientes cujas idades variaram de um dia a oito meses. Seus pesos corporais estavam compreendidos entre 2 e $8 \mathrm{~kg}$. Esses pacientes foram operados com circulaçăo extracorpórea, hipotermia profunda $\left(18^{\circ} \mathrm{C}\right.$ no nasofaringe) e parada circulatória total, por períodos de 20 a 60 minutos $^{2,12,31}$.

Os demais pacientes ( 80$)$, embora com peso corporal inferior a $10 \mathrm{~g}$, constituíram um grupo selecionado, nāo comparável ao anterior em idades e lesōes e foram operados com perfusão contínua e baixo fluxo arterial, durante a realização do tempo operatório principal ${ }^{33}$.

\section{Protocolos utilizados}

A adequada realização da CEC nesses pequenos pacientes requer o conhecimento não apenas da doença, mas da fisiologia e da imaturidade estrutural e metabólica de certos órgăos, como cérebro, fígado e rins, por exemplo.

\section{Circuito e perfusato}

O circuito utilizado é relativamente simples. Deve ser o mais curto possivel, para reduzir o volume inicial do perfusato.

A estrutura básica do circuito que usamos é demonstrada no Quadro 1.

Utilizamos um pequenos segmento de linha de 1/4 de polegada, intercalado como shunt, entre as linhas arterial e venosa, que permite a lenta recirculação do perfusato durante os períodos de parada circulatória ${ }^{11}$ 26
QUADRO 1

COMPOSICYÃO E ESTRUTURA DO CIRCUITO EXTRACORPOREO

CIRCUITO EXTRACORPÓREO

(Neonatos e Lactentes)

OXIGENADOR

Shiley S070-S

Macchi "Baby"

TUBOS DE 1/4" (Curtos)

Linha Arterial

Linha Venosa

"Shunt" A-V

Linhas Aspiradoras (2)

CARDIOTOMIA COM FILTRO

\section{QUADRO 2 \\ COMPOSICYÃO DO PERFUSATO USADO PARA NEONATOS E LACTENTES}

\section{PERFUSATO}

Concentrado de Hemácias

Plasma Fresco

$300 \mathrm{ml}$

Heparina 300 a $400 \mathrm{ml}$

Gluconato de Cálcio $5 \mathrm{mg} / 100 \mathrm{ml}$

Bicarbonato de Sódio 8,4\% $0,1 \mathrm{~g} / 100 \mathrm{ml}$ Metilprednisolona (Solu-Medrol)

.............................. $300 \mathrm{mg}$

Os oxigenadores são de bolhas, porém especificamente desenhados para crianças, não sendo miniaturas dos oxigenadores usados para adultos. Atualmente, estamos ajustando nossos protocolos para o uso de oxigenadores de membrana capilar, exclusivamente para este tipo de pacientes.

O fluxômetro de gás é de coluna longa, bastante sensível e capaz de injetar pequenos fluxos de oxigênio, propiciando fácil controle da oxigenação e, conseqüentemente, do $\mathrm{pO}_{2}$. Năo misturamos $\mathrm{CO}_{2}$ ao gás injetado, em nenhuma circunstância.

O perfusato é crítico, tanto no seu volume quanto na composição ${ }^{23},{ }^{30}$ (Quadro 2).

O volume inicial é de 600 a $700 \mathrm{ml} \mathrm{e}$ a hemodiluiçăo é feita, basicamente, com plasma fresco congelado. As quantidades de concentrado de hemácias podem variar, dependendo do hematócrito inicial do paciente. Procuramos obter um hematócrito em perfusão de 20 a $25 \%$.

A água para o paciente provém da hidratação intraoperatória e da diluição das drogas administradas.

Esse rigor na composiçăo do perfusato $e$ administração de cristalóides objetiva manter as vantagens da 
ELIAS, D. O.; SOUZA, M. H. L.; LACERDA, B. S.; FAGUNDES, F. E. S.; LINO, F. J. S.; TIRABOSHI, M. - Injúria orgânica da circulação extracorpórea nos trés primeiros meses de vida. Rev. Bras. Cir. Cardiovasc., 5(1): 1-8, 1990.

hemodiluição e preservar a pressão coloidosmótica do perfusato ${ }^{26}$, principal causa de edema durante a CEC. A hemodiluiçăo com soluçōes cristalóides é mal tolerada pelos neonatos e produz edema intersticial importante que leva a complicações pulmonares e seqüestro progressivo no terceiro espaço, de difícil reversão.

Essa tendência ao desenvolvimento de edema com a hemodiluição é maior nas crianças que usam prostaglandinas no pré-operatório. Essas drogas aumentam, consideravelmente, a permeabilidade capilar.

Antes do início da perfusão, o perfusato é recirculado, levemente aquecido $\left(36^{\circ} \mathrm{C}\right)$, e o seu $\mathrm{pH}$ verificado e neutralizado, se necessário.

\section{Anticoagulação}

Administramos heparina ( $3 \mathrm{mg} / \mathrm{kg}$ de peso) e mantemos o TCA automatizado entre 480 e 600 segundos, monitorizado a intervalos regulares, durante todo o procedimento.

A neutralização da heparina ao final da perfusăo é feita na proporçāo de 1:1 a no máximo 1:1.5 de sulfato de protamina, também monitorizado pelo TCA ${ }^{32}$.

\section{Fluxos e condução da perfusáo}

Iniciamos a perfusão com o perfusato a $36^{\circ} \mathrm{C}$ e estabilizamos a hemodinâmica da perfusão, sem desviar volume do paciente para o oxigenador, o que, invariavelmente, produz bradicardia ou fibrilação, que prejudica a perfusão miocárdica antes do clampeamento aórtico e das medidas específicas de proteção.

A perfusão subendocárdica é muito dependente da contraçāo miocárdica e da pressāo final dentro do ventrículo. Se o coraçăo se esvazia e pára rapidamente, a resistência vascular se eleva no leito coronário e o miocárdio pode sofrer injúria.

O fluxo de perfusão é de $200 \mathrm{ml} / \mathrm{kg} / \mathrm{min}$., mantido até atingirmos a temperatura de $18^{\circ} \mathrm{C}$ no nasofaringe e 20 a $22^{\circ} \mathrm{C}$ no reto, quando, então, promovemos a parada circulatória ${ }^{4,34}$. Fluxos altos de perfusão protegem melhor o organismo neonatal, dificultam ou impedem a vasoconstriçāo, resfriam mais homogeneamente e reduzem consideravelmente a produção de ácido lático, que é outro fator de agressão miocárdica, durante a fase de resfriamento.

\section{Monitorizaçáo}

Os principais parâmetros de monitorização são fluxo arterial, gasometria arterial, temperatura do nasofaringe, retal e da água do permutador de calor. O leito vascular, nesses pequenos pacientes, é muito distensível e com- placente, tem grande reatividade e a cânula arterial é necessariamente fina. Isto torna a pressão arterial sempre baixa e mau indicador de qualquer evento da perfusăo.

As temperaturas do nasofaringe e retal, sua evolução e gradientes sāo melhores indicadores da adequação da perfusāo, que quaisquer outros parâmetros.

A temperatura do nasofaringe reflete as temperaturas do cérebro, miocárdio, rins e outros órgãos nobres, enquanto a temperatura retal reflete, principalmente, os órgãos abdominais e massas musculares. Os gradientes entre essas duas temperaturas nos indicam a homogeneidade do resfriamento e a adequaçăo da perfusão.

\section{Controle do equilíbrio ácido-básico}

A hipotermia é um estado de profunda anormalidade da fisiologia do ser humano. A experiência tem demonstrado que o metabolismo tissular aeróbico e as funçōes enzimáticas celulares são mais eficientes em um regime de alcalose. Assim, mantemos a alcalose respiratória da perfusão hipotérmica sem adição de $\mathrm{CO}_{2}$ ou outros recursos.

$\mathrm{O} \mathrm{pH}$ se eleva cerca de 0,0147 e $\circ \mathrm{pCO}_{2}$ cerca de $4 \%$ para cada grau de redução da temperatura. Aceitamos esse regime de alcalose progressiva, conhecido como "alfa-stat", para o manuseio do equilibrio ácidobásico.

Assim, para uma temperatura de $18^{\circ} \mathrm{C}$, nos parece adequado um $\mathrm{pH}$ de 7,8 e um $\mathrm{pCO}_{2}$ em torno de 20 a $23 \mathrm{mmHg}$. Isso, como vimos, é importante para o metabolismo geral do paciente e, principalmente, para o metabolismo miocárdico ${ }^{6,25}$.

Com o reaquecimento, voltamos à estratégia habitual para o equilibrio ácido-basico em normotermia.

\section{Proteção do miocárdio}

O miocárdio do neonato e do lactente tem características próprias que o diferenciam do miocárdio adulto. Essas diferenças são relacionadas à sua estrutura, função e metabolismo ${ }^{15},{ }^{21}$. Além disso, o miocárdio neonatal tolera melhor a hipóxia e responde pior às drogas inotrópicas.

Essas diferenças podem estar relacionadas com a insuficiente proteção que a cardioplegia cristalóide usada para adultos confere ao miocárdio infantil ${ }^{18}$.

Nossa experiência tem demonstrado que o miocárdio neonatal e lactente é melhor protegido pela combinaçăo de hipotermia tópica e cardioplegia sangüínea com cálcio normal, em dose única, e está de acordo com a experiência de numerosos autores ${ }^{5,7,21}$. 
ELIAS, D. O.; SOUZA, M. H. L.; LACERDA, B. S.; FAGUNDES, F. E. S.; LINO, F. J. S.; TIRABOSHI, M. - Injúria orgânica da circulaçăo extracorpórea nos três primeiros meses de vida. Rev. Bras. Cir. Cardiovasc., 5(1): 1-8, 1990.

QUADRO 3

CARACTERISTICAS DA SOLUÇĀO DE CARDIOPLEGIA SANGÜINEA UTILIZADA EM NEONATOS E LACTENTES

\section{CARDIOPLEGIA SANGÜÍNEA}

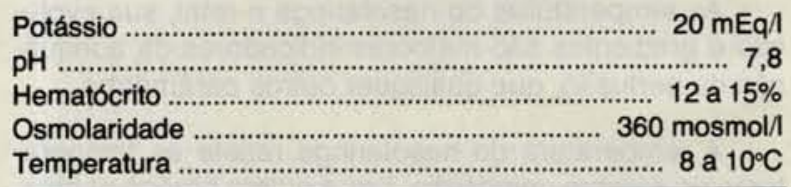

Nossa solução de cardioplegia tem as características mostradas no Quadro 3.

\section{Parada circulatória}

Para essas operaçōes em neonatos preferimos a parada circulatória total em temperatura de $18^{\circ} \mathrm{C}$ no nasofaringe. Ao fazermos a parada circulatória, o perfusato é lentamente circulado através do shunt entre as linhas arterial e venosa. $\mathrm{O} \mathrm{pH}$ é verificado e corrigido, se necessário.

Respeitamos o limite de 50 minutos para a parada circulatória, só excepcionalmente atingindo uma hora. Quando necessário, retornamos à perfusão por cinco minutos e fazemos novo período de parada circulatória, o que ocorre apenas excepcionalmente.

Todas as evidências da literatura mostram que uma parada circulatória de 50 minutos é bastante segura ${ }^{31}$. 33, 34 .

A discussão gira sempre em torno do grau de proteção cerebral durante o período de parada da circulação. Se o resfriamento é induzido lenta e progressivamente, com um perfusato alcalótico e sem evidências de vasoconstrição, a proteção do sistema nervoso central é sistematicamente adequada e a incidência de complicaçōes neurológicas não é maior que a verificada na perfusão contínua convencional.

\section{Injúrias da perfusão: mecanismos de complicaçōes}

De um modo geral, podemos afirmar que o resultado de uma operação intracardiaca nessas pequenas crianças depende, fundamentalmente, da condução adequada e simultânea dos quatro principais eventos na sala de operaçōes: a) procedimento anestésico; b) correção intracardiaca; c) proteção do miocárdio; d) perfusão (CEC).

É estimado que aproximadamente $80 \%$ do resultado final está na dependência desse conjunto de eventos, cujas complicaçōes mais graves raralmente podem ser revertidas no pós-operatório, por mais sofisticado que possa ser.
Desse conjunto de eventos, o que tem mais chance de contribuir para o desenvolvimento de complicaçōes, com grande frequeência mal toleradas, é a perfusão.

A maioria das complicaçōes é conseqüência de alguns tipos de injúria, que podem ocorrer durante a perfusão e que podem se potencializar reciprocamente, tornando a sua soma total superior à tolerância daqueles pequenos organismos.

O único veículo de "comunicaçăo" entre a perfusão e o organismo do paciente é o sangue, ao qual toda a injúria do procedimento é imposta.

Agrupamos as injúrias segundo o seu mecanismo de produção, cientes de que podem ocorrer isolada ou simultaneamente. Analisamos quatro tipos principais de injúrias: 1) injúria mecânica; 2) injúria físico-química; 3) injúria metabólica; 4) injúria térmica.

\section{Injúria mecânica}

A injúria mecânica ocorre em função das forças mecânicas a que o sangue está submetido durante os procedimentos da CEC e que diferem totalmente das forças do bombeamento normal do sistema circulatório, bem como diferem substancialmente as superfícies sobre as quais atuam aquelas forças ${ }^{35,}{ }^{36}$.

Essa ação mecânica, ou a injúria mecânica, pode ser produzida em diversos pontos dos oxigenadores e do circuito e a sua expressão clínica, bem conhecida, é a hemólise.

A hemólise tem um significado mais amplo que a simples produção de hemoglobinúria. A injúria não atinge seletivamente as hemácias. Assim, a ocorrência de hemólise significa que os leucócitos, as plaquetas, o fibrinogênio e as demais proteínas do plasma ligadas à coagulação sofreram injúria mecânica, juntamente com as hemácias.

Isso explica a freqüente associação que observamos, na prática, entre o aparecimento de hemólise, ou hemoglobinúria, e distúrbios da coagulação.

Essa injúria mecânica de que tratamos pode ser produzida nos seguintes pontos do circuito extracorpóreo: dispersor de oxigênio dos oxigenadores, câmara de oxigenação dos oxigenadores de bolhas, bomba arterial, filtros, conectores e aspiradores, sendo estes últimos os maiores produtores da injúria mecânica.

\section{Injúria físico-química}

A injúria de natureza físico-química não é tão fácil de identificar e está ligada, principalmente, a certas características dos materiais de que são fabricados os equipamentos com os quais o sangue entra em contato ${ }^{13}$. 
ELIAS, D. O.; SOUZA, M. H. L.; LACERDA, B. S.; FAGUNDES, F. E. S.; LINO, F. J. S.; TIRABOSHI, M. - Injúria orgânica da circulação extracorpórea nos três primeiros meses de vida. Rev. Bras. Cir. Cardiovasc., 5(1): 1-8, 1990.

Apesar da sua relativa inércia química e, mesmo, biológica por fenómenos nāo bem esclarecidos, esses materiais podem ativar o fator XII (Fator Hageman) do plasma sangüíneo. Este, por seu turno, pode ativar os sistemas das cininas, da coagulaçāo, da fibrinólise, ou do complemento ${ }^{14,17,20,28}$.

Um ou vários desses sistemas, quando ativados, liberam certas substâncias do plasma e de alguns tipos de células que, no seu conjunto, produzem uma reação inflamatória geral do organismo, com agregação celular e plaquetária, vasoconstriçāo, aumento da permeabilidade capilar e lesões da membrana celular ${ }^{19}$.

Clinicamente, suas conseqüências se traduzem pelo aparecimento, em diversos graus e associaçōes, de: edema generalizado, disfunçāo pulmonar, acidose metabólica, discrasias sangüíneas, insuficiência renal, síndrome pós-perfusão e menor resistência às infecções.

\section{Injúria metabólica}

Os neonatos que se submetem à cirurgia, ou têm cianose, ou insuficiência cardiaca descompensada, condiçōes em que, ou coexiste acidose metabólica, ou o seu desenvolvimento é muito fácil. A sua reserva de buffers é muito limitada e insuficiente para compensar a própria acidose.

Como todas as substâncias usadas para o perfusato são ácidas, incluindo-se o sangue estocado e seus derivados, o seu $\mathrm{pH}$ deve ser criteriosamente tamponado a todo momento, visto que a acidose metabólica pode ocorrer com muita facilidade. Isso, como já mencionamos, prejudica o metabolismo celular geral e miocárdico, podendo produzir injúria ainda antes do clampeamento aórtico ${ }^{3,10,24,25}$.

Certas drogas, como o nitroprussiato, metabolizadas pelo fígado, são mal metabolizadas durante a hipotermia e seus catabolitos podem causar reaçōes tóxicas, de diagnóstico muito difícil dentro do contexto da CEC.

A esse tipo de injúria, chamamos metabólica e procuramos evitá-la com atenção ao metabolismo, sua redução pela hipotermia e manutençãoo de condições adequadas à função enzimática celular (alcalose).

\section{Injúria térmica}

Estamos habituados a considerar as trocas térmicas do permutador de calor sob a perspectiva da calorimetria simples, o que não acontece, na realidade.

O permutador de calor dos oxigenadores funciona como um sistema termodinâmico complexo. Há movimento dos líqüidos, água e sangue, dos dois lados da interface de trocas térmicas, a fluxos diferentes e existe uma fonte permanente de calor no sistema, que é a resistência elétrica da bomba de água.

Para um lactente de $5 \mathrm{~kg}$ de peso, por exemplo, o fluxo de perfusão é de $1 \mathrm{l} / \mathrm{min}$., enquanto o fluxo da bomba de água que aquece o sangue é de cerca de $20 \mathrm{l} / \mathrm{min}$. Portanto, existe uma relação de 1:20 entre os dois fluxos envolvidos nas trocas de calor, durante o reaquecimento.

Existe uma interface entre a água e o sangue, que é a superfície de trocas do permutador de calor. Outra interface existe entre o sangue e os tecidos, que é o revestimento endotelial dos capilares. $E$ uma outra interface, entre os tecidos e as cavidades nasofaringe ou do reto, que é a membrana celular. O gradiente correto a ser medido e respeitado é entre a temperatura da água e do sangue arterial, como podemos entender.

Assim, temos observado, no reaquecimento, que a temperatura do sangue arterial se eleva rapidamente e pode, mesmo, superar a temperatura da água que o aquece, por causa da diferença entre os fluxos. Se não estivermos atentos à monitorização da temperatura do sangue arterial e apenas medirmos o nasofaringe ou o reto, não vamos detectar o superaquecimento do sangue, que pode ultrapassar os $42 \mathrm{ou}$, mesmo, os $45^{\circ} \mathrm{C}$. Isso ocorre sempre, se usarmos a água a $42^{\circ} \mathrm{C}$ de rotina.

O superaquecimento do sangue pode produzir a desnaturação das proteínas do plasma, especialmente do fibrinogênio e outras ligadas à coagulação, bastate lábeis. Isso produz discrasias sangüíneas de difícil diagnóstico e controle.

Pode, ainda, coexistir dano térmico aos elementos figurados do sangue, o que outra vez será traduzido pelo aparecimento de hemólise e hemoglobinúria.

Essa injúria não é rara em neonatos e só pode ser evitada com a adequada monitorização das temperaturas do sangue e da água do permutador de calor.

Nossa incidência de hemorragias reduziu-se consideravelmente, quando passamos a detectar e impedir essa injúria térmica ao sangue, durante a perfusão.

\section{RESULTADOS}

Vamos analisar suscintamente nossa experiência com a parada circulatória total, que nos permitiu evoluir até os protocolos atuais. Estes 110 casos incluem os neonatos e lactentes até $8 \mathrm{~kg}$ de peso. Todas as operaçōes habitualmente realizadas nessa faixa etária estão representadas, exceto a correção anatômica da TGA pela operação de Jatene.

Como a experiência foi acumulada ao longo de um tempo, durante o qual os protocolos foram modificados, ela serve apenas como amostragem geral dos procedimentos de CEC (Tabela 1). 
ELIAS, D. O.; SOUZA, M. H. L.; LACERDA, B. S.; FAGUNDES, F. E. S.; LINO, F. J. S.; TIRABOSHI, M. - Injúria orgânica da circulação extracorpórea nos três primeiros meses de vida. Rev. Bras. Cir. Cardiovasc., 5(1): 1-8, 1990.

TABELA 1

MORTALIDADE E PRINCIPAIS COMPLICAÇÓES OBSERVADAS

\begin{tabular}{|c|c|c|c|}
\hline \multicolumn{4}{|c|}{$\begin{array}{c}\text { HIPOTERMIA PROFUNDA E PARADA CIRCULATORIA } \\
\text { TOTAL }\end{array}$} \\
\hline 6 & $N ?$ & $\%$ & $\angle C 70(\%)$ \\
\hline Total de casos & 110 & $100 \%$ & - \\
\hline Total de óbitos & 29 & $26,4 \%$ & $22-31$ \\
\hline \multicolumn{4}{|c|}{ COMPLICAÇÓES IMPORTANTES } \\
\hline Hemorragias (Reoperaçōes) & 12 & $10,9 \%$ & $8-15$ \\
\hline Cegueira transitória & 2 & $1,8 \%$ & $1-4$ \\
\hline Convulsōes & 6 & $5,5 \%$ & $3-9$ \\
\hline Hipocalcemia & 2 & $1,8 \%$ & $1-4$ \\
\hline Hipoglicemia & 3 & $2,7 \%$ & $1-5$ \\
\hline
\end{tabular}

A maioria das complicaçóes observadas pode ser atribuída à perfusão, embora, em muitos casos, essa correlação seja mais difícil de estabelecer.

As complicações relacionadas na Tabela 1, referem-se apenas aos pacientes que sobreviveram às ope- raçōes. Assim, certos casos de insuficiência renal, por exemplo, ocorreram com alguns pacientes que foram a óbito e não estăo ali relacionadas.

Diversos pacientes desta experiência apresentaram mais de uma complicaçăo.

A causa de óbito mais comum foi o baixo débito cardíaco.

\section{CONCLUSŌES}

A perfusão para neonatos e lactentes, por qualquer técnica, pode ser considerada um exercício contínuo de paciência e de criteriosa atençăo aos detalhes, por mais insignificantes que possam parecer.

A seleção do equipamento e material a ser utilizado, seu preparo, montagem e a condução da pertusão devem levar em conta os aspectos particulares da fisiologia desses pequenos organismos e sua resposta à injúria. 0 mínimo de trauma, o respeito às necessidades metabólicas e, sobretudo, a prevençăo de graus importantes de injúria significam, com freqüência, a diferença entre o sucesso e o insucesso desses procedimentos.

RBCCV 44205-101

ELIAS, D. O.; SOUZA, M. H. L.; LACERDA, B. S.; FAGUNDES, F. E. S.; LINO, F. J. S.; TIRABOSHI, M. - Organic injury of extracorporeal circulation in the first three months of life. Rev. Bras. Cir. Cardiovasc., $5(1): 1-8,1990$.

ABSTRACT: Newborns and small infants require special procedures of extracorporeal circulation for open heart surgery, which must be based on their specific physiological and metabolic patterns, as well as on the response to deviations of their homeostasis. The authors reviewed the protocols of cardiopulmonary bypass utilized during 100 procedures with total circulatory arrest and discuss some mechanisms by which the blood components may be damaged or injured during perfusion. Mechanical injury of solid blood components and plasma proteins are discussed; the activation of certain plasmatic systems as the coagulation, fibrinolytic and complement by the Hageman factor, which can produce a generalized inflammatory reaction is also identified; deviations of acid-base balance by excessive lactic acid production may impair enzymatic and cellular functions which can cause injury of a metabolic nature. Thermal injury may also be produced by overheating blood during rewarming if the dynamic nature of heat exchangers function is not adequately monitored. The conclusion is that neonatal perfusion represents a continuing exercise of attention to detail and must be performed with a profound respect to their specific physiology, metabolism and response to injury.

DESCRIPTORS: extracorporeal circulation, children

\section{REFERÊNCIAS BIBLIOGRÁFICAS}

1 BACKER, C. L.; ILBAWI, M. N.; OHTAKE, S.; DE LEON, S.; MUSTER, A. J.; PAUL, M. H.; BENSON Jr., D. W.; IDRISS, F.S. - Transposition of the great arteries: a comparison of results of the Mustard procedure versus the arterial switch. Ann. Thorac. Surg., 48: 10-14, 1989.
2 BARRAT-BOYES, B. G.; NEUTZE, J. M.; SEELYE, E. R.; SIMPSON, M. - Complete correction of cardiovascular malformations in the first year of life. Progr. Cardiovasc. Dis., 15: 229-234, 1972.

3 BAUM, D.; DILLARD, D. H.; MOHRI, H.; CRAWFORD, E. W. - Metabolic aspects of deep surgical hypothermia in infancy. Pediatrics, 42: 93-105, 1968. 
ELIAS, D. O.; SOUZA, M. H. L.; LACERDA, B. S.; FAGUNDES, F. E. S.; LINO, F. J. S.; TIRABOSHI, M. - Injúria orgânica da circulação extracorpórea nos três primeiros meses de vida. Rev. Bras. Cir. Cardiovasc., 5(1): 1-8, 1990.

4 BERRYESSA, R.; HYDRICK, D.; MCCORMICK, J.; TYNDALL, C. M.; PETERSON, D.; TORNABENE, M.; TORNABENE, S.; WILLIAMS, W.; PAPPAS, G.; CAMP. BELL, D.; CLARKE, D. R. - Refinements in infant/pediatric perfusion. J. Extracorp. Technol., 18: 140-144, 1986.

5. BUCKBERG, G. D. - Strategies and logic of cardioplegic delivery to prevent, avoid, and reverse ischemic and reperfusion damage. J. Thorac. Cardiovasc. Surg., 93: 127-139, 1987.

6. BUCKBERG, G. D.; STEEED, D.; BECKER, H.; ROSENKRANZ, E. R. - Myocardial protection during paediatric cardiac surgery. In: MARCELLETTI, C.; ANDERSON, R. H.; BECKER, A. E.; CORNO, A.; Di CARLO, D.; MAZZERA, E. (eds.) Paediatric Cardiology. London, Churchill Livingstone, 1986. p. 39-52.

7 BULL, C.; COOPER, J.; STARK, J. - Cardioplegic protection of the child's heart. J. Thorac. Cardiovasc. Surg., 88: 287-293, 1984.

8 CASTAÑEDA, A. R.; LAMBERTI, J.; SADE, R. M.; WILLIAMS, R. G.; NADAS, A. S. - Open-heart surgery during the first three months of life. J. Thorac. Cardiovasc. Surg., 68: 719-723, 1974.

9. CLARKE, D. R. - Pediatric perfusion: an evolving science. J. Extracorp. Technol., 21: 18-20, 1989.

10. DAVIES, G. C.; SOBEL, M.; SALZMAN, E. W. - Elevated plasma fibrinopeptide $A$ and thromboxane $B 2$ levels during cardiopulmonary bypass. Circulation, 61: 808-814, 1980.

11. DE LEVAL, M. - Perfusion techniques for paediatric cardiac surgery. In: TAYLOR, K. M. Cardiopulmonary bypass: principles and management. Baltimore, Williams \& Wilkins, 1986. p. 115-123.

12. DILLARD, D. H.; MOHRI, H.; HESSEL II, E. A.; ANDERSON, H. N.; NELSON, R. J.; CRAWFORD, E. W.; MORGAN, B. C.; WINTERSCHEID, L. C.; MERENDINO, K. A. - Corrections of total anomalous pulmonary venous drainage in infancy utilizing deep hypothermia with total circulatory arrest. Circulation, 105 (Supl. 1): 35-36, 1967.

13. EDMUNDS Jr., L. H. \& ADDONIZIO Jr., V. P. - Platelet physiology during cardiopulmonary bypass. In: UTLEY, J. R. (ed.) Pathophysiology and techniques of cardiopulmonary bypass. Baltimore, Williams \& Wilkins, 1982. p. 106-119.

14. FAYMONVILLE, M. E.; DEBY-DUPONT, G.; LARBUISSON, R.; DEBY, C.; BODSON, L.; LIMET, R.; LAMY, M. - Prostaglandin E2, prostacyclin, and thromboxane changes during nonpulsatile cardiopulmonary bypass in humans. J. Thorac. Cardiovasc. Surg., 91 (6): 858-866, 1986.

15. FRIEDMAN, W. F. - Physiological properties of the developing heart. In: MARCELLETTI, C.; ANDERSON, R. H.; BECKER, A. E.; CORNO, A.; Di CARLO, D.; MAZ-
ZERA, E. (eds.) Paediatric Cardiology. London, Churchill Livingstone, 1986. p. 3-12.

16. GRAHAM, G. - A personal review of over 20 years' experience in ECC in infants. In: HAGL, S.; KLOVEKORN, W. P.; MAYR, N.; SEBENING, F. Thirty years of extracorporeal circulation. Munich, Carl Gerber, 1984. p. 65-73.

17. HAMMERSCHMIDT, D. E.; STRONCEK, D. F.; BOWERS, T. K. - Complement activation and neutropenia during cardiopulmonary bypass. J. Thorac. Cardiovasc. Surg., 86: 845-957, 1983.

18. KEMPSFORD, R. D. \& HEARSE, D. J. - Protection of the immature heart: temperature-dependent beneficial or detrimental effects of multidose crystalloid cardioplegia in the neonatal rabbit heart. J. Thorac. Cardiovasc. Surg., 99: 269-279, 1990.

19. KIRKLIN, J. K. - Low flow pertusion and the damaging effects of cardiopulmonary bypass. In: MARCELLETTI, C.; ANDERSON, R. H.; BECKER, A. E.; CORNO, A.; Di CARLO, D.; MAZZERA, E. (eds.). Paediatric Cardiology, London, Churchill Livingstone, 1986. p. 53-58.

20. KIRKLIN, J. K.; WESTABY, S.; BLAKSTONE, E. H.; KIRKLIN, J. W.; CHENOWETH, D. E.; PACIFICO, A. D. - Complement and the damaging effects of cardiopulmonary bypass. J. Thorac. Cardiovasc. Surg., 86: 845-857, 1983.

21. LAKS, H.; MILLIKEN, J.; HASS, G. - Myocardial protection in the neonatal heart. In: MARCELLETTI, C.; ANDERSON, R. H.; BECKER, A.E.; CORNO, A.; Di CARLO, D.; MAZZERA, E. (eds.) Paediatric Cardiology, London, Churchill Livingstone, 1986. p. 13-27.

22. LAKS, H. - The arterial switch procedure for the neonate: coming of age. Ann. Thorac. Surg., 48: 3-4, 1989.

23. LINDBERG, H.; OVRUM, E.; HOLEN, E. A.; ABDELNOOR, M.; SOMMERFELT, S. C. - Changes in colloid oncotic pressure during and after cardiopulmonary bypass. Pertusion, 3: 47-53, 1988.

24. MARATH, A.; MAN, W.; TAYLOR, K. M. - Paediatric extracorporeal priming procedures: does low temperature priming promote histamine release? Perfusion, 3: 287-293, 1988.

25. MILNE, E. G.; ELLIOTT, M. J.; PEARSON, D. T.; HOL DEN, M. P.; ORSKOV, H.; ALBERTI, K. G. M. M. The effect on intermediary metabolism of open-heart surgery with deep hypothermia and circulatory arrest in infants of less than 10 kilograms body weight: a preliminary study. Perfusion, 1: 29-40, 1986.

26. NICOLAS, F.; BRUNIAUX, J.; PLANCHE, C.; BINET, J. $P$. - Recent techniques of cardiopulmonary bypass in infancy. In: HAGL, S.; KLOVEKORN, W. P.; MAYR, N.; SEBENING, F. (eds.). Thirty years of extracorporeal circulation. Munich, Carl Gerber, 1984. p. 75-85. 
ELIAS, D. O.; SOUZA, M. H. L.; LACERDA, B. S.; FAGUNDES, F. E. S.; LINO, F. J. S.; TIRABOSHI, M. - Injúria orgânica da circulação extracorpórea nos trés primeiros meses de vida. Rev. Bras. Cir. Cardiovasc., 5(1): 1-8, 1990.

27. NORWOOD, W. I.; DOBELL, A. R.; FREED, M. D.; KIRKLIN, J. W.; BLAKSTONE, E. H. - Intermediate results of the arterial switch repair: a 20th Institution study. J. Thorac. Cardiovasc. Surg., 96: 854-863, 1988.

28. OEVEREN, W. \& WILDEVUUR, C. R. H. - Blood compatibility of cardiopulmonary bypass circuits. Pertusion, 2: 237-244, 1987.

29. PLANCHE, C.; BRUNIAUX, J.; LACOUR-GAYET, F.; KACHANER, J.; BINET, J. P.; SIDI, D.; VILLAIN, E. Switch operation for transposition of the great arteries in neonates: a study of 120 patients. J. Thorac. Cardiovasc. Surg., 96: 354-363, 1988.

30. SADE, R. M.; STROUD, M. R.; CRAWFORD Jr., F. A.; KRATZ, J. M.; DEARING, J. P.; BARTLES, D. M. A prospective randomized study of hydroxyethyl starch, albumin, and lactated Ringer's solution as priming fluid for cardiopulmonary bypass. J. Thorac. Cardiovasc. Surg., 89: 713-722, 1985.

31. THARION, J.; JOHNSON, D. C.; CELEMANER, J. M. Profound hypothermia with circulatory arrest: nine years' clinical experience. J. Thorac. Cardiovasc. Surg., 84: 66-72, 1982.
32. UTLEY, J. R. - Early development of cardiopulmonary bypass. Pertusion, 1: 1-14, 1986.

33. WELLS, F. C.; COGHILL, S.; CAPLAN, H. L.; LINCOLN, C.; KIRKLIN, J. W. - Duration of circulatory arrest does influence the psychological develpment of children after cardiac operations in early life. J. Thorac. Cardiovasc. Surg., 86: 823-831, 1983.

34. WILLIAMS, G. D.; SEIFEN, A. B.; LAWSON, N. W.; NORTON, J. B.; READINGER, R. I.; DUNGAN, T. W.; CALLAWAY, J. K. - Pulsatile pertusion versus conventional high-flow nonpulsatile perfusion for rapid core cooling and rewarming of infants for circulatory arrest in cardiac operations. J. Thorac. Cardiovasc. Surg., 78: 667-674, 1979.

35. WRIGHT, G. - Blood cell trauma. In: TAYLOR, K. M. (ed.). Cardiopulmonary bypass: principles andmanagement. Baltimore, Williams \& Wilkins, 1986. p. 249-276.

36. WRIGHT, G. - The hydraulic power outputs of pulsatile and nonpulsatile cardiopulmonary bypass pumps. Pertusion, 3: 251-262, 1988. 